\title{
Impacto da remoção de serapilheira sobre a comunidade de artrópodes edáficos em plantios abandonados de Corymbia citriodora
}

\author{
Litter removal impact on an edaphic arthropod community in abandoned \\ Corymbia citriodora plantations
}

\author{
Rodrigo Camara ${ }^{\mathrm{I}}$, Vinicius Duncan Silva ${ }^{\mathrm{II}}$, Maria Elizabeth Fernandes Correia ${ }^{\mathrm{III}}$, Dora Maria Villela ${ }^{\mathrm{IV}}$
}

\begin{abstract}
Resumo
Experimentos de manipulação podem contribuir para a compreensão do papel ecológico da serapilheira em ecossistemas florestais. No entanto, há poucos estudos desta natureza. Os efeitos da remoção da camada de serapilheira (RS) foram avaliados sobre a comunidade de artrópodes do solo em dois plantios abandonados de Corymbia citriodora, em diferentes estágios de regeneração natural de espécies nativas de Mata Atlântica. Armadilhas de queda foram instaladas aleatoriamente em 5 parcelas $(5 \times 20 \mathrm{~m}$ ) de RS e controle (CT), no plantio com 19 anos de idade (P19: estágio menos avançado; maior participação da serapilheira de eucalipto) e plantio com 42 anos (P42: estágio mais avançado de regeneração; maior participação da serapilheira de espécies nativas), nas estações chuvosa e seca, na Reserva Biológica União, RJ, Brasil. A maioria dos grupos taxonômicos apresentou inibição da abundância com a RS, no P19 e P42. De uma maneira geral, Diptera, Poduromorpha, Pseudoscorpionida, Symphypleona e larvas de Coleoptera foram os principais grupos impactados negativamente pela RS. Por outro lado, alguns grupos foram favorecidos pela RS, principalmente Entomobryomorpha e Formicidae. A RS diminuiu a riqueza total, uniformidade e diversidade de artrópodes edáficos em ambos os plantios. Contudo, este efeito negativo foi mais importante no P19, no qual também ocorreu a diminuição da abundância total e riqueza média. O maior fechamento do dossel provavelmente minimizou o impacto negativo da RS sobre os artrópodes edáficos no P42.
\end{abstract}

Palavras-chave: Eucalipto; Fauna do solo; Mata Atlântica; Ciclagem de nutrientes

\begin{abstract}
Manipulation experiments can contribute to understand the ecological role of litter in forest ecosystems. However, there are few studies of this nature. We evaluated the effects of the litter layer removal (RS) on the soil arthropod community in two abandoned plantations of Corymbia citriodora at different stages of the Atlantic Rainforest natural regeneration. Pitfall traps were randomly installed in plots $(5 \times 20 \mathrm{~m})$ of RS and control (CT) in a 19-year old plantation (P19: less advanced stage of natural regeneration of Atlantic Rainforest species; higher contribution of eucalypt in litterfall) and a 42-year old plantation (P42: more advanced stage of regeneration; higher contribution of native species in litterfall), in rainy season and dry season at 'União Biological Reserve', RJ state, Brazil. RS inhibited the abundance of the most taxonomic groups, both in P19 and P42. In general, Diptera, Poduromorpha, Pseudoscorpionida, Symphypleona, and larvae of Coleoptera were the most negatively impacted groups by RS. In contrast, RS favored some other groups, principally Entomobryomorpha and Formicidae. Total richness, evenness and diversity were lower in RS in both plantations. However, this negative effect was stronger in the P19, where RS also decreased total abundance and average richness. The higher canopy closure probable minimized the negative impact of RS on the soil arthropods in P42.
\end{abstract}

Keywords: Eucalyptus; Soil fauna; Atlantic Rainforest; Nutrient cycling

\footnotetext{
${ }^{I}$ Engenheiro Agrônomo, Dr., Pós-Doutorando pela Universidade Federal Rural do Rio de Janeiro, Rod. BR 465, Km 07, s/n, Zona Rural, CEP 23890-000, Seropédica (RJ), Brasil. Bolsista da CAPES. rcamara73@gmail.com (ORCID: 0000-0002-89253260)

II Biólogo, MSc., Universidade Estadual do Norte Fluminense Darcy Ribeiro, Av. Alberto Lamego, 2000, Parque Califórnia, CEP 28013-600, Campos dos Goytacazes (RJ), Brasil. vini.duncan@gmail.com (ORCID: 0000-0001-7102-3582)

III Bióloga, Dra ., Pesquisadora da Embrapa Agrobiologia, Rod. BR-465, Km, 7, RJ, CEP 23891-000, Seropédica (RJ), Brasil. ecorreia@cnpab.embrapa.br (ORCID: 0000-0003-1919-6659)

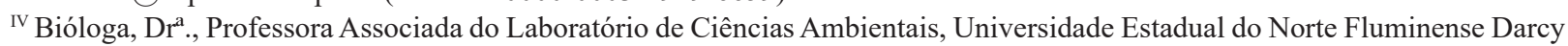
Ribeiro, Av. Alberto Lamego, 2000, Parque Califórnia, CEP 28013-600, Campos dos Goytacazes (RJ), Brasil. dora@uenf.br (ORCID: 0000-0002-3507-0186)
} 


\section{Introdução}

O desflorestamento em áreas tropicais visa ao atendimento de variadas atividades humanas, como a obtenção de madeira, expansão de fronteiras agropecuárias e instalação de loteamentos e indústrias. Os plantios com espécies florestais exóticas de rápido crescimento, como eucaliptos, proporcionam a obtenção de recursos que incluem a madeira, o que concorre para diminuir a pressão sobre as florestas nativas (ALENCAR et al., 2011). Além disto, os plantios de eucalipto podem funcionar como catalisadores da regeneração natural de espécies nativas (ONOFRE; ENGEL; CASSOLA, 2010), desde que haja áreas de floresta nativa próximas para fornecer propágulos (ONOFRE; ENGEL; CASSOLA, 2010; ALENCAR et al., 2011). Porém, a comunidade de espécies vegetais nativas que caracteriza a regeneração natural sob os plantios de eucalipto comumente apresenta menores valores de riqueza e/ou diversidade, na comparação com fragmentos de floresta nativa adjacentes (ALEM; PAVLIS, 2012). Este mesmo resultado foi observado com relação à regeneração de espécies nativas em plantios de eucalipto com diferentes idades, que estão abandonados desde 1996 e foram estabelecidos na Reserva Biológica União, antes que esta unidade de conservação fosse instituída em 1998, a qual abriga um importante remanescente de Mata Atlântica, no Estado do Rio de Janeiro (EVARISTO; BRAGA; NASCIMENTO, 2011).

Este padrão provavelmente é o reflexo da presença de compostos alelopáticos na serapilheira foliar dos eucaliptos, cujo impacto é negativo para a germinação de sementes e o estabelecimento de plântulas (ZHANG; FU, 2009). Este material orgânico também apresenta menor qualidade química, devido à elevada razão $\mathrm{C} / \mathrm{N}$ e ao baixo conteúdo nutricional, na comparação com a serapilheira produzida por espécies florestais nativas (GAMA-RODRIGUES et al., 2008). Desta maneira, a serapilheira produzida por eucaliptos não favorece a biota edáfica decompositora e contribui pouco para a ciclagem de nutrientes. Tal panorama ilustra a importância da camada de serapilheira disposta na superfície do solo florestal como componente ecológico que influencia não só a germinação e o estabelecimento de plântulas, como também a dinâmica da ciclagem de nutrientes (IBÁÑEZ; SCHUPP, 2002). Assim, a serapilheira é capaz de influenciar a composição de espécies em ecossistemas florestais (SAYER, 2006; CIERJACKS et al., 2007).

Com isto, experimentos de manipulação da camada de serapilheira em plantios de eucalipto com regeneração de espécies nativas podem contribuir para a compreensão do papel deste material orgânico em diferentes aspectos ecológicos, tal como a sua influência sobre a comunidade de artrópodes do solo. Este tópico é de extrema importância, uma vez que os referidos organismos atuam de maneira decisiva no processo de ciclagem de nutrientes (WANG; RUAN; WANG, 2009), além do fato de não haver dados desta natureza.

O presente estudo objetivou: (1) avaliar o efeito da presença/ausência da camada de serapilheira sobre a estrutura e composição da comunidade de artrópodes edáficos, em dois plantios abandonados de eucalipto com regeneração de espécies nativas de Mata Atlântica; e (2) identificar os grupos taxonômicos que compõem esta comunidade que são mais ou menos afetados pela remoção da camada de serapilheira. Foi testada a hipótese de que a remoção da camada de serapilheira diminui a complexidade da estrutura e altera a composição da comunidade de artrópodes edáficos em plantios abandonados de eucalipto, em diferentes estágios de regeneração de Mata Atlântica, em comparação com a presença da camada de serapilheira, na Reserva Biológica União, RJ.

\section{Material e métodos}

\section{Caracterização da área de estudo}

A Reserva Biológica União localiza-se sob as coordenadas $22^{\circ} 27^{\prime} 30^{\prime \prime}$ 'S e 42 $02^{\prime} 15^{\prime \prime} \mathrm{W}$, e distribui-se em parte dos municípios Rio das Ostras, Casimiro de Abreu e Macaé, no Rio de Janeiro. Esta unidade de conservação federal apresenta 3.126 ha de área total, dos quais 220 ha referem-se a 47 plantios abandonados de eucalipto em diferentes idades, com diferentes estágios de regeneração natural de espécies nativas de Mata Atlântica. Entretanto, grande parte da área (aproximadamente $2.400 \mathrm{ha}$ ) corresponde a áreas de floresta que pertence ao domínio da Mata Atlântica, denominada Floresta Ombrófila Densa de terras baixas e submontanas. Segundo Köppen, o clima da região é classificado como Aw, isto é, tropical úmido com inverno seco (ALVARES et al., 2013). A temperatura média anual é de $22^{\circ} \mathrm{C}$ e a precipitação total anual é de $2.337 \mathrm{~mm}^{\text {ano }}{ }^{-1}$, a qual se concentra em grande parte (89\% do total) entre os meses de outubro e abril (CAMARA; CORREIA; VILLELA, 2012). 
A comunidade de artrópodes edáficos foi estudada em dois plantios de Corymbia citriodora (Hook.) K. D. Hill \& L. A. S. com 19 anos (P19) e 42 anos (P42), os quais foram instalados em 1991 e 1968, respectivamente. O espaçamento de plantio no P19 e P42 é de 1,5 x 3,0 m e 3,0 x 3,0 m, enquanto a área total é de 7,20 ha e 11,44 ha, respectivamente. A regeneração natural de espécies nativas de Mata Atlântica, que se iniciou quando os plantios foram abandonados em 1996, foi considerada incipiente em ambos os plantios (EVARISTO; BRAGA; NASCIMENTO, 2011). Contudo, de acordo com estes autores, tal processo foi considerado mais avançado no sub-bosque de P42, no qual as espécies arbóreas Xylopia sericea A. St. - Hil. (Annonaceae), Eugenia supraaxillaris Spring (Myrtaceae) e Cupania oblongifolia Mart. (Sapindaceae) compreendem a maioria das espécies (64\%), ao passo que Myrsine coriacea (Sw.) R. Br. ex Roem. \& Schult. (Myrsinaceae) é a única espécie arbórea nativa na regeneração natural no sub-bosque de P19. Em ambos os plantios, o solo foi classificado como Argissolo Vermelho Amarelo distrófico latossólico (MIRANDA; CANELLAS; NASCIMENTO, 2007).

\section{Amostragem da comunidade de artrópodes edáficos}

Em agosto de 2004, foram instaladas 10 parcelas (5 m x 20 m) em P19 e P42 para a realização de outros trabalhos nas áreas (EVARISTO; BRAGA; NASCIMENTO, 2011). Em cinco destas parcelas, a camada de serapilheira tem sido manipulada até o presente momento, por meio da remoção manual com ancinhos de jardinagem, mensalmente, constituindo o tratamento de remoção de serapilheira (RS). Nas demais parcelas, que constituem o controle (CT), a camada de serapilheira tem sido mantida. As folhas de Corymbia citriodora apresentam elevada participação relativa na serapilheira aportada ao solo em ambos os plantios, mas esta contribuição é maior em P19 (96\% do total da serapilheira foliar), na comparação com o P42 (46\%) (CAMARA et al., 2018b).

A coleta dos artrópodes edáficos ocorreu por meio da instalação de duas armadilhas de queda (pitfall traps) em cada uma das parcelas, resultando em 10 armadilhas por tratamento, em cada plantio de eucalipto. Estas armadilhas, que foram enterradas no solo com a abertura rente à superfície, consistiram em recipientes plásticos $(500 \mathrm{~mL}, 9 \mathrm{~cm}$ diâmetro, $11 \mathrm{~cm}$ altura) que foram preenchidos parcialmente com aproximadamente $150 \mathrm{ml}$ de uma solução de ácido acetilsalicílico a 3\%. As armadilhas foram cobertas com uma bandeja descartável de alumínio $(830 \mathrm{~mL}, 19 \mathrm{~cm}$ diâmetro, $4,5 \mathrm{~cm}$ altura) sustentada por estacas de madeira, para evitar a entrada de água da chuva e serapilheira.

Após o período de sete dias consecutivos em que permaneceram no campo, nas estações chuvosa (janeiro/2010) e seca (agosto/2010), as armadilhas foram removidas e conduzidas ao Laboratório de Fauna do Solo da Embrapa Agrobiologia. Em seguida, o conteúdo das armadilhas foi transferido para placas de Petri, com o auxílio de pissete (água destilada), e armazenado em frascos (10 mL) contendo álcool a $70 \%$, até o momento das avaliações.

Análises dos dados da comunidade de artrópodes edáficos

Cada armadilha foi considerada uma unidade experimental. Os organismos edáficos foram quantificados e identificados em grandes grupos (ordem, classe, superfamília, família). Em função da facilidade de identificação, a família Formicidae foi considerada um grupo taxonômico à parte da ordem Hymenoptera, ao passo que a infraordem Isoptera foi considerada um grupo taxonômico à parte da ordem Blattodea. A classe Collembola foi subdividida em três grupos taxonômicos: Entomobryomorpha, Poduromorpha e Symphypleona. Os grupos taxonômicos que apresentaram participação relativa inferior a $5 \%$ foram reunidos sob a denominação "Outros".

Os valores de abundância ( $\mathrm{Ab}$, número de indivíduos por armadilha por dia, ind $\left.\operatorname{arm}^{-1} \mathrm{~d}^{-1}\right)$ total e para os grupos; riqueza total (Rt, número total de grupos taxonômicos); e riqueza média (Rm, média do número de grupos calculada entre as unidades experimentais) foram estimados. Os índices de uniformidade de Pielou (U, distribuição do número de indivíduos por grupos) e de diversidade de Shannon-Wiener (H’) foram calculados segundo Odum (1988). 
As possíveis alterações observadas na abundância dos grupos taxonômicos como resposta à RS também foram avaliadas por meio do cálculo do índice $\mathrm{V}$ ou índice de modificação (WARDLE; PARKINSON, 1991). Este índice indica o grau de distúrbio ou estabilidade da abundância dos grupos de artrópodes, como resposta ao tratamento ou condição ambiental testada (CUNHA NETO et al., 2012; LIMA et al., 2017). Para tanto, empregou-se a seguinte equação:

$$
\mathrm{V}=((2 \mathrm{x} / \mathrm{x}+\mathrm{y})-1)
$$

Em que: $\mathrm{x}=$ abundância dos grupos na RS; $\mathrm{y}=$ abundância dos grupos no CT (referência).

Por meio do valor deste índice, que varia de -1 a +1 , o efeito do tratamento sobre a abundância pode ser classificado em categorias de inibição, estimulação ou sem alteração (Tabela 1) (modificado por WARDLE, 1995).

\section{Tabela 1 - Classificação da inibição ou estimulação da abundância dos artrópodes edáficos como resposta à remoção da serapilheira, de acordo com o índice de modificação V (modificado por WARDLE, 1995).}

Table 1-Ranking of inhibition or stimulation of the abundance of the edaphic arthropods in response to the litter removal, according to the change index V (modified by WARDLE, 1995).

\begin{tabular}{lc}
\hline \multicolumn{1}{c}{ Categoria } & Valor do índice $\mathbf{~}$ \\
\hline Inibição extrema & $\mathrm{V}<-0,67$ \\
Inibição moderada & $-0,33>\mathrm{V}>-0,67$ \\
Inibição leve & $-0,05>\mathrm{V}>-0,33$ \\
Sem alteração & $-0,05<\mathrm{V}<0,05$ \\
Estimulação leve & $0,05<\mathrm{V}<0,33$ \\
Estimulação moderada & $0,33<\mathrm{V}<0,67$ \\
Estimulação extrema & $\mathrm{V}>0,67$ \\
\hline
\end{tabular}

Os resultados de $\mathrm{Ab}$ total e de grupos, bem como de $\mathrm{Rm}$, foram testados segundo ANOVA e as médias dos tratamentos (RS; CT) em cada plantio de eucalipto, em ambas as estações climáticas, foram comparadas com o teste não paramétrico de Mann-Whitney a 5\% de significância, com o auxílio da versão 7.0 do software Statistica. Construiu-se um gráfico de análise de componentes principais com o objetivo de identificar os grupos taxonômicos que apresentaram maior correlação com as condições testadas (tratamentos, plantios, estações climáticas). Para tanto, consideraram-se apenas os grupos taxonômicos cujos valores de abundância média apresentaram coeficientes de correlação maiores do que $0,70 \%$, com um dos eixos principais (eixo 1 ou eixo 2). Além disto, realizou-se a análise de agrupamento hierárquico, com a finalidade de se identificar possíveis padrões de dissimilaridade entre as situações testadas (tratamentos, plantios, estações climáticas). Neste sentido, considerou-se a abundância média de todos os grupos taxonômicos, para a obtenção de um dendrograma de dissimilaridade desenvolvido pela distância de Bray-Curtis, baseado no método de grupos pareados. Ambas as análises multivariadas foram realizadas por meio da versão $2.17 \mathrm{c}$ do software PAST.

\section{Resultados e discussão}

Nos plantios de eucalipto estudados, foi amostrado um total de 8.914 indivíduos que se distribuíram em 28 grupos taxonômicos. Destes, os mais representativos foram Entomobryomorpha (38\% do total), Formicidae (22\%), Diptera (12\%), Acari (7\%), Orthoptera (6\%) e Symphypleona (5\%), os quais contribuíram com $90 \%$ dos indivíduos de toda a comunidade. O restante da comunidade (10\%) foi representado pelo conjunto denominado Outros, que incluiu Araneae, Auchenorrhyncha, Blattodea, Chilopoda, Coleoptera (formas larvais e adultos), Diplopoda, Heteroptera, Hymenoptera, Isopoda, Isoptera, Mantodea, Neuroptera, Poduromorpha, Pseudoscorpionida, Psocoptera, Sternorrhyncha, Thysanoptera, Trichoptera (larvas e adultos), larvas de Diptera e larvas de Lepidoptera. 
Os grupos Poduromorpha e Pseudoscorpionida foram capturados apenas no CT, no P19 (estação chuvosa). Ambos os grupos taxonômicos comumente ocorrem em ecossistemas menos perturbados ou em recuperação (FERREIRA et al., 2017; LIMA et al., 2017). Este fato indica a presença de um ambiente edáfico mais adequado para a comunidade de artrópodes do solo como um todo (PEREIRA et al., 2013), condição que foi proporcionada pela manutenção da camada de serapilheira. Por outro lado, três grupos taxonômicos se restringiram ao tratamento de RS: Chilopoda (P42, estação chuvosa), Neuroptera (P19, estação seca) e Trichoptera (P42, estação seca).

Não houve um padrão claro de influência do tratamento de serapilheira sobre a participação relativa da maioria dos grupos taxonômicos predominantes, na comunidade de artrópodes edáficos. Entretanto, de uma maneira geral observou-se que no CT foram maiores as participações relativas de Diptera (ambos os plantios de eucalipto, nas duas estações climáticas), enquanto as de Entomobryomorpha foram maiores na RS (P19, em ambas as estações climáticas, e P42, na estação chuvosa) (Figura 1).

Figura 1 - Participação relativa (\%) dos grupos taxonômicos de artrópodes do solo predominantes no controle (CT) e remoção da serapilheira (RS), nos plantios com 19 e 42 anos de idade (P19 e P42, respectivamente) de Corymbia citriodora, nas estações chuvosa e seca, Reserva Biológica União, RJ.

Figure 1 - Relative participation (\%) of the predominant taxonomic groups of the soil arthropods community in control (CT) and litter removal (RS), at 19 and 42 years old Corymbia citriodora plantations (P19 and P42, respectively), in rainy and dry season, 'União' Biological Reserve, RJ state.

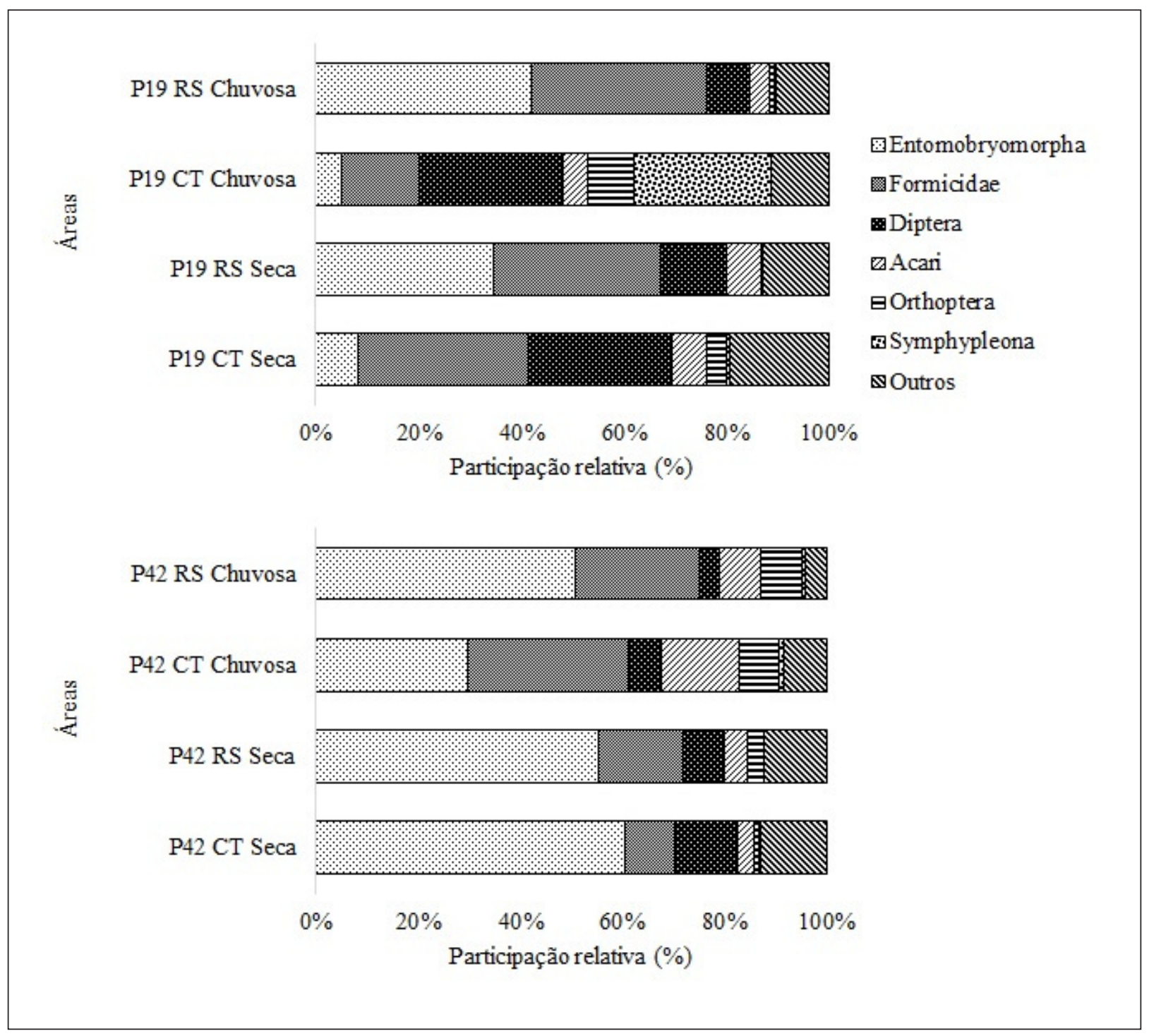


Os valores de abundância total e riqueza média no P19 foram significativamente menores na RS, quando comparado com o CT, na estação chuvosa (Tabela 2). Este mesmo padrão foi verificado nos dois plantios de eucalipto, para a riqueza total (ambas as estações climáticas), diversidade (P19 em ambas as estações climáticas; P42 na estação chuvosa) e uniformidade (P19 na estação seca; P42 na estação chuvosa).

Tabela 2 - Atributos estruturais da comunidade de artrópodes do solo no controle (CT) e remoção da serapilheira (RS), nos plantios com 19 e 42 anos de idade (P19 e P42, respectivamente) de Corymbia citriodora, nas estações chuvosa e seca, Reserva Biológica União, RJ.

Table 2 - Structural attributes of the soil arthropods community in control (CT) and litter removal (RS), at 19 and 42 years old Corymbia citriodora plantations (P19 and P42, respectively), in rainy and dry seasons, 'União' Biological Reserve, RJ state.

\begin{tabular}{|c|c|c|c|c|c|c|c|c|}
\hline \multirow{3}{*}{ Atributos estruturais } & \multicolumn{4}{|c|}{ Estação chuvosa } & \multicolumn{4}{|c|}{ Estação seca } \\
\hline & \multicolumn{2}{|c|}{ P19 } & \multicolumn{2}{|c|}{ P42 } & \multicolumn{2}{|c|}{ P19 } & \multicolumn{2}{|c|}{ P42 } \\
\hline & CT & RS & CT & RS & CT & RS & CT & RS \\
\hline \multirow[t]{2}{*}{$\mathrm{Ab}$} & $21,07 \mathrm{~A}$ & $8,97 \mathrm{~B}$ & 23,51 & 28,01 & 5,91 & 4,30 & 17,63 & 17,93 \\
\hline & $(3,06)$ & $(1,16)$ & $(3,26)$ & $(4,04)$ & $(0,96)$ & $(1,12)$ & $(2,11)$ & $(1,67)$ \\
\hline $\mathrm{Rm}$ & $11 \mathrm{~A}$ & $7 \mathrm{~B}$ & 12 & 10 & 6 & 6 & 10 & 10 \\
\hline Rt & 21 & 15 & 22 & 18 & 16 & 15 & 18 & 16 \\
\hline $\mathrm{U}$ & 0,53 & 0,56 & 0,56 & 0,51 & 0,69 & 0,63 & 0,51 & 0,57 \\
\hline $\mathrm{H}^{\prime}$ & 2,32 & 2,18 & 2,52 & 2,11 & 2,76 & 2,46 & 2,14 & 2,27 \\
\hline
\end{tabular}

Em que: Os valores de abundância total $(\mathrm{Ab})$ estão seguidos pelo respectivo erro padrão, entre parênteses. Valores com diferença significativa na mesma linha, entre diferentes tratamentos dentro do mesmo plantio de eucalipto e estação climática, são seguidos por letras diferentes. Rm: riqueza média; Rt: riqueza total; $\mathrm{U}$ : uniformidade; H': diversidade.

Desta maneira, a classificação taxonômica empregada no presente estudo permitiu identificar que a RS impactou negativamente a estrutura da comunidade de artrópodes edáficos nos dois plantios de eucalipto considerados. Este mesmo padrão foi anteriormente verificado para a abundância/densidade de artrópodes edáficos em florestas tropicais no Panamá (SAYER; TANNER; LACEY, 2006; ASHFORD et al., 2013) e florestas temperadas nos EUA (CASTRO; WISE, 2009; OBER; DEGROOTE, 2011). Determinados organismos edáficos são forçados a migrar para áreas onde não houve remoção da camada de serapilheira, $o$ que diminui a riqueza com o manejo de remoção (SAYER; TANNER; LACEY, 2006). Contudo, Ashford et al. (2013) não observaram diminuição da diversidade como resposta à remoção de serapilheira.

No presente estudo, resposta divergente (maiores valores dos atributos estruturais na RS) ocorreu apenas para a uniformidade no P19 (estação chuvosa) e no P42 (estação seca), assim como para a diversidade no P42 (estação seca) (Tabela 2). Alguns grupos apresentaram diminuição dos valores de abundância como um reflexo da RS em ambos os plantios de eucalipto. Entretanto, esta resposta foi mais importante no P19 em comparação com o P42. Tal resultado foi verificado no P19 para os herbívoros Diptera (CT: 5,89 $\pm 0,89$ ind $\operatorname{arm}^{-1} \mathrm{~d}^{-1}$ RS: $0,76 \pm 0,18$, na estação chuvosa; CT: 1,64 $\pm 0,39$, RS: $0,56 \pm 0,10$, na estação seca), Orthoptera (CT: $1,90 \pm 0,56$, RS: $0,07 \pm 0,03$, na estação chuvosa; CT: $0,21 \pm 0,06$, RS: $0,01 \pm 0,01$, na estação seca) e Auchenorrhyncha (CT: 0,46 $\pm 0,10$, RS: $0,13 \pm$ 0,05 , na estação seca). O mesmo padrão ocorreu no P19 para os micrófagos/saprófagos Symphypleona (CT: 5,66 $\pm 2,25$, RS: $0,03 \pm 0,02$, na estação chuvosa) e no P42 para os saprófagos/predadores larvas de Coleoptera (CT: $0,17 \pm 0,05$, RS: $0,04 \pm 0,03$, na estação chuvosa).

O impacto negativo da RS nos dois plantios de eucalipto foi corroborado pelos resultados do índice $\mathrm{V}$, indicando um elevado percentual de grupos cuja abundância passou por algum tipo de inibição, quando comparado com os percentuais verificados para as categorias de estimulação e ausência de alteração em função deste manejo da serapilheira, em ambas as estações climáticas (Tabela 3). De uma maneira geral, entre as categorias de inibição da abundância de grupos em virtude da RS, prevaleceu a inibição extrema, tanto em P19 quanto em P42. 
Tabela 3 - Distribuição percentual (\%) dos grupos taxonômicos de artrópodes do solo nas categorias do índice V, em função da remoção da serapilheira (RS), nos plantios com 19 e 42 anos de idade (P19 e P42, respectivamente) de Corymbia citriodora, nas estações chuvosa e seca, Reserva Biológica União, RJ.

Table 3 - Percentage distribution (\%) of soil arthropods taxonomic groups by index V categories, in the litter removal (RS) at 19 and 42 years old Corymbia citriodora plantations (P19 and P42, respectively), in rainy and dry seasons, 'União' Biological Reserve, RJ state.

\begin{tabular}{|c|c|c|c|c|}
\hline \multirow{3}{*}{ Categoria do índice $V$} & \multicolumn{2}{|c|}{ P19 (RS) } & \multicolumn{2}{|c|}{ P42 (RS) } \\
\hline & Estação chuvosa & Estação seca & Estação chuvosa & Estação seca \\
\hline & \multicolumn{4}{|c|}{$\%$} \\
\hline Inibição extrema & 50 & 30 & 30 & 11 \\
\hline Inibição moderada & 18 & 15 & 9 & 0 \\
\hline Inibição leve & 14 & 15 & 26 & 39 \\
\hline $\begin{array}{l}\text { Total de grupos (\%) com } \\
\text { algum tipo de inibição }\end{array}$ & 82 & 60 & 65 & 50 \\
\hline Estimulação leve & 0 & 0 & 9 & 17 \\
\hline Estimulação moderada & 5 & 10 & 4 & 17 \\
\hline Estimulação extrema & 9 & 20 & 4 & 11 \\
\hline $\begin{array}{l}\text { Total de grupos (\%) com } \\
\text { algum tipo de estimulação }\end{array}$ & 14 & 30 & 17 & 45 \\
\hline Sem alteração & 4 & 10 & 18 & 5 \\
\hline Total de grupos (\%) & 100 & 100 & 100 & 100 \\
\hline
\end{tabular}

No entanto, mais uma vez observou-se que este impacto negativo da RS foi proporcionalmente mais importante no P19 (82\% e 60\% dos grupos apresentaram algum tipo de inibição da abundância, nas estações chuvosa e seca, respectivamente), em relação ao P42 (65\% e 50\% dos grupos com inibição da abundância, nas estações chuvosa e seca, respectivamente). Desta maneira, o índice V se configurou como uma importante ferramenta que salientou a resposta da comunidade de artrópodes a diferentes condições do solo, fato este que corroborou os resultados obtidos por outros estudos previamente realizados em diferentes ecossistemas (CUNHA NETO et al., 2012; LIMA et al., 2017).

Organismos pertencentes a todas as guildas tróficas foram capturados em ambos os plantios de eucalipto, nas duas estações climáticas, independentemente do tratamento de serapilheira. Porém, entre os saprófagos e os micrófagos prevaleceu a categoria de inibição extrema, enquanto que entre os predadores prevaleceu a inibição leve, como resposta à RS no P19 e no P42, nas estações chuvosa e seca. A camada de serapilheira na superfície do solo constitui recurso alimentar para os artrópodes saprófagos e para os microrganismos decompositores. Estes, por sua vez, são recurso alimentar para os artrópodes predadores e os micrófagos, respectivamente. Portanto, a remoção da serapilheira influencia negativamente a comunidade de artrópodes do solo de maneira direta e indireta, em ecossistemas florestais (SAYER, 2006). O desfavorecimento de diferentes guildas tróficas indica que o ambiente edáfico é menos estável (FERREIRA et al., 2017), sendo que esta condição, que prejudica a dinâmica da decomposição de serapilheira e, dessa forma, a ciclagem de nutrientes (FRASSON et al., 2016), está associada à remoção da camada de serapilheira.

Apenas organismos pertencentes a dois grupos apresentaram aumento da abundância com a RS: os micrófagos/saprófagos Entomobryomorpha no P19 (CT: 1,09 $\pm 0,11$ ind $\operatorname{arm}^{-1} \mathrm{~d}^{-1}$ RS: 3,77 $\pm 0,77$, estação chuvosa), e os saprófagos/predadores Formicidae no P42 (CT: 1,74 $\pm 0,50$ ind arm ${ }^{-1}$ $\mathrm{d}^{-1}$, RS: $2,94 \pm 0,43$, estação seca). O aumento populacional de Entomobryomorpha é uma função do incremento do conteúdo de matéria orgânica no solo (ROVEDDER et al., 2009). Entretanto, o conteúdo de matéria orgânica no solo certamente foi diminuído com a RS, no presente estudo. Portanto, acredita- 
se que a maior abundância deste grupo no referido tratamento de serapilheira tenha ocorrido em função da elevada mobilidade / atividade de Entomobryomorpha, na interface serapilheira-solo. Grupos que apresentam esta característica tendem a ser numericamente mais capturados por meio de armadilhas de queda, em relação àqueles de mobilidade limitada (GONÇALVES; PEREIRA, 2012). Apesar disto, a remoção de serapilheira não alterou a abundância do grupo Collembola, que abrange os representantes de Entomobryomorpha, em florestas tropicais no Panamá, embora tenha diminuído a abundância de Formicidae, quando comparado com o controle (ASHFORD et al., 2013).

No caso de Formicidae, os organismos deste grupo podem apresentar elevada abundância mesmo com a RS, como também foi observado em plantios de Pinus elliottii Engelm, Pinus palustris Miller e Pinus taeda L. nos EUA (OBER; DEGROOTE, 2011). Este resultado é consequência da elevada mobilidade e capacidade de localização e captura de alimento pelas formigas, em áreas onde não ocorreu a remoção da serapilheira (LINDSAY; CUNNINGHAM, 2009).

Com relação à análise dos componentes principais, a variabilidade dos dados explicada foi de $46,94 \%$ no eixo 1 e $43,53 \%$ no eixo 2, o que correspondeu a 90,47\% da variância entre os tratamentos de serapilheira nos plantios de eucalipto, nas estações climáticas. Esta análise demonstrou que houve o agrupamento das condições de CT e RS no P19 e P42 na estação seca, e RS em P19 e P42 na estação chuvosa, que se situaram na porção esquerda do eixo 1 e na porção inferior do eixo 2 (Figura 2).

Figura 2 - Diagrama de ordenação obtido pela análise de componentes principais da abundância de grupos de artrópodes edáficos nas condições de CT (controle) e RS (remoção de serapilheira) nos plantios com 19 e 42 anos de idade (P19 e P42, respectivamente) de Corymbia citriodora, nas estações chuvosa e seca, Reserva Biológica União, RJ. Aran: Araneae; Blatt: Blattodea; Isop: Isopoda; Isopt: Isoptera; l. Cole: larvas de Coleoptera; Mant: Mantodea; Podu: Poduromorpha; Pseu: Pseudoscorpionida; Psoc: Psocoptera; Ster: Sternorrhyncha; Symp: Symphypleona.

Figure 2 - Ordinational diagram obtained by the principal component analysis (PCA) for the abundance of the soil arthropod groups in control (CT) and litter removal (RS), at 19 and 42 years old Corymbia citriodora plantations (P19 and P42, respectively), in the rainy and dry seasons, 'União' Biological Reserve, RJ state. Aran: Araneae; Blatt: Blattodea; Isop: Isopoda; Isopt: Isoptera; 1. Cole: Coleoptera’s larvae; Mant: Mantodea; Podu: Poduromorpha; Pseu: Pseudoscorpionida; Psoc: Psocoptera; Ster: Sternorrhyncha; Symp: Symphypleona.

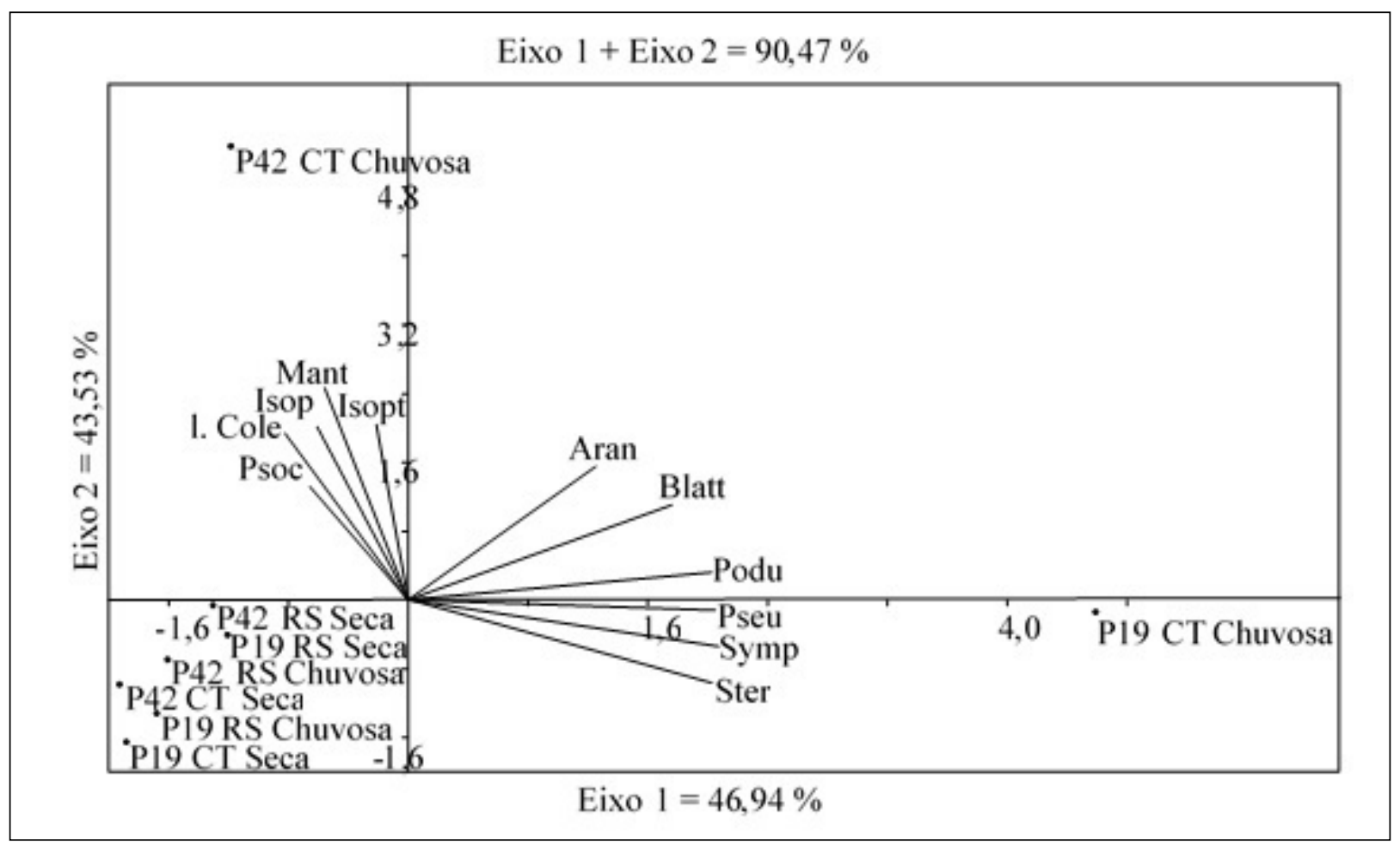


Em contrapartida, CT em P19 na estação chuvosa e CT em P42 nesta mesma estação apresentaramse completamente separados entre si. Contudo, a condição CT em P19 na estação chuvosa mostrou maior dissimilaridade entre as demais condições, visto que foi a única que se situou na porção direita do eixo 1 (valores positivos), enquanto que todas as outras se localizaram na porção esquerda do mesmo eixo (valores negativos). Este padrão sugeriu que a estação climática pode ter influenciado no efeito da remoção/presença da camada de serapilheira sobre a comunidade de artrópodes edáficos. Variações sazonais afetam a ocorrência/abundância de determinados grupos taxonômicos (CAMARA; CORREIA; VILLELA, 2012; CAMARA et al., 2017) e guildas tróficas destes organismos, principalmente no que se refere a micrófagos, predadores e herbívoros, o que altera os índices de diversidade e uniformidade da comunidade de artrópodes edáficos (MANHÃES et al., 2013).

A Figura 2 também indicou que Araneae, Blattodea, Poduromorpha, Pseudoscorpionida, Symphypleona e Sternorrhyncha apresentaram maior correlação com o CT no P19 (estação chuvosa) (Figura 2). Por outro lado, Isopoda, Isoptera, Mantodea, Psocoptera e larvas de Coleoptera mostraram maior correlação com o CT no P42 (estação chuvosa). Portanto, a manutenção da camada de serapilheira na superfície do solo favoreceu a abundância dos referidos grupos, nas duas áreas estudadas. Nenhum grupo taxonômico correlacionou-se com a RS nos plantios de eucalipto.

A camada de serapilheira minimiza as variações drásticas nas condições de umidade e temperatura no solo (WOLKOVICH, 2010) e, consequentemente, a sua remoção é desfavorável para a comunidade de artrópodes do solo. Até mesmo a diminuição da espessura desta camada pode impactar negativamente a abundância destes organismos, em florestas tropicais (YANG; WARREN; ZOU, 2007; SAYER et al., 2010). O efeito tamponante positivo da serapilheira sobre as condições microclimáticas do solo florestal, e portanto sobre os artrópodes edáficos, pode ser considerado até mesmo mais importante do que o seu conteúdo nutricional (BULTMAN; UETZ, 1982). Tal fato foi atestado por Gill (1969), que verificou que não houve diferença na abundância de artrópodes do solo ao comparar o efeito de adição de serapilheira natural e a substituição desta por serapilheira artificial sem valor nutritivo (fibras de poliéster), em florestas temperadas nos EUA.

A manutenção da camada de serapilheira foi positiva para a comunidade de artrópodes edáficos, em ambos os plantios de eucalipto. Desta maneira, foi corroborada a hipótese de que a remoção da camada de serapilheira diminui a complexidade da estrutura e altera a composição da comunidade de artrópodes edáficos em plantios abandonados de eucalipto, em diferentes estágios de regeneração de Mata Atlântica, em comparação com a presença da camada de serapilheira, na Reserva Biológica União, RJ. Contudo, a magnitude deste impacto foi maior no P19. Nesta área, a complexidade da estrutura da comunidade arbórea é muito menor, em comparação com o P42 (EVARISTO; BRAGA; NASCIMENTO, 2011). Portanto, acreditase que o maior efeito negativo da remoção da serapilheira sobre a comunidade de artrópodes edáficos no P19 tenha ocorrido em função da maior abertura do dossel, que é formado apenas por indivíduos de Corymbia citriodora. Por outro lado, o maior fechamento do dossel no P42 provavelmente amenizou este impacto.

Em áreas de floresta tropical, em que o dossel é mais aberto, a incidência de luz na superfície do solo é maior (KEELING; PHILLIPS, 2007). Esta condição, por sua vez, incorre na formação de um microclima edáfico desfavorável para os artrópodes do solo, os quais são submetidos a altas temperaturas e baixo conteúdo de água no solo (MARTIUS et al., 2004). Por este motivo, a densidade de artrópodes edáficos é menor em áreas de floresta tropical com dossel mais aberto, quando comparada com áreas onde o dossel é mais fechado (MORÓN-RÍOS; HUERTA-LWANGA, 2006). No entanto, tal hipótese não foi testada no presente estudo, uma vez que a temperatura e o conteúdo de água do solo não foram avaliados. Sugere-se que um novo estudo desta natureza seja realizado, para investigar se a magnitude do efeito observado no P19 apresenta continuidade ou não, em função do fechamento do dossel que ocorre ao longo do desenvolvimento ecossistêmico.

De acordo com o diagrama de agrupamento hierárquico, ocorreu a formação de dois grandes grupos, por meio dos quais se individualizou os plantios estudados (Figura 6). Dentro do grande grupo representado por P19, houve a separação de dois subgrupos: um formado pela comunidade de artrópodes do solo no P19 CT na estação chuvosa, e outro formado pela reunião de P19 CT na estação seca e P19 RS nas estações seca e chuvosa. O grande grupo referente ao P42, por sua vez, subdividiuse em um subgrupo representado por P42 CT na estação chuvosa e um subgrupo que reuniu P42 CT na estação seca e P 42 RS nas estações seca e chuvosa. 
Figura 3 - Dendrograma de dissimilaridade de acordo com a abundância média dos grupos taxonômicos de artrópodes edáficos nas condições de CT (controle) e RS (remoção de serapilheira), nos plantios com 19 e 42 anos de idade (P19 e P42, respectivamente) de Corymbia citriodora, nas estações chuvosa e seca, Reserva Biológica União, RJ.

Figure 3 - Dendrogram of dissimilarity according to the average abundance of the abundance of the soil arthropod groups in control (CT) and litter removal (RS), at 19 and 42 years old Corymbia citriodora plantations (P19 and P42, respectively), in the rainy and dry seasons, 'União' Biological Reserve, RJ state.

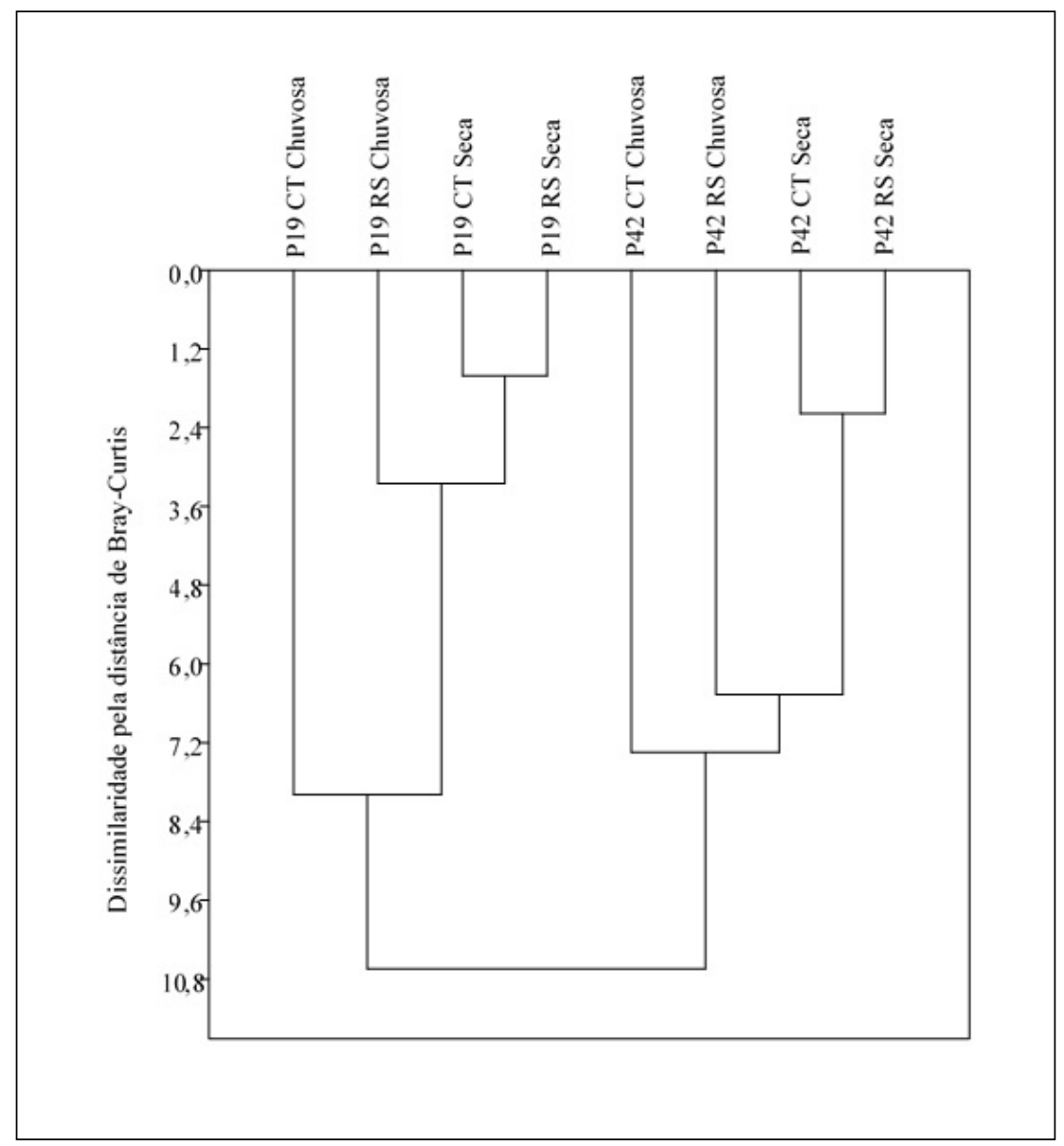

A Figura 3 demonstrou a elevada dissimilaridade entre os plantios estudados, com relação à abundância dos grupos taxonômicos que constituem a comunidade de artrópodes edáficos, independentemente da manipulação da serapilheira e da estação climática. Tal padrão sugeriu que a complexidade da estrutura da comunidade arbórea, que é maior no P42, exerceu influência relativamente maior sobre a abundância de artrópodes edáficos, quando comparado com a manipulação da serapilheira, apesar desta hipótese não ter sido testada no presente estudo. Em um estudo realizado previamente, onde se considerou apenas o controle, constatou-se que os valores de abundância média de Acari e Formicidae (estação chuvosa), além de Entomobryomorpha e da comunidade (estação seca) foram significativamente maiores no P42, na comparação com o P19 (CAMARA; CORREIA; VILLELA, 2012). Maiores valores de abundância de artrópodes do solo também foram encontrados em áreas em estágio mais avançado de regeneração natural de espécies nativas de Mata Atlântica, no município de Pinheiral, estado do Rio de Janeiro, independentemente da estação climática (CAMARA et al., 2018a). 


\section{Conclusões}

Corroborou-se a hipótese de que a remoção da camada de serapilheira diminui a complexidade da estrutura e altera a composição da comunidade de artrópodes edáficos em plantios abandonados de eucalipto, em diferentes estágios de regeneração de Mata Atlântica, em comparação com a presença da camada de serapilheira, na Reserva Biológica União, RJ.

Diptera, larvas de Coleoptera, Poduromorpha, Pseudoscorpionida e Symphypleona foram os principais grupos impactados negativamente pela remoção de serapilheira, além de Araneae, Auchenorrhyncha, Blattodea, Isopoda, Isoptera, Mantodea, Orthoptera, Psocoptera e Sternorrhyncha. Por outro lado, principalmente Entomobryomorpha e Formicidae, além de Chilopoda, Neuroptera e Trichoptera, foram de alguma maneira favorecidos pela remoção de serapilheira.

\section{Agradecimentos}

Os autores agradecem ao Laboratório de Ciências Ambientais (LCA) da Universidade Estadual do Norte Fluminense Darcy Ribeiro (UENF), pelo apoio logístico; à Fundação de Amparo à Pesquisa do Estado do Rio de Janeiro (FAPERJ), pela bolsa de doutorado fornecida ao primeiro autor, na Pós-graduação em Ecologia e Recursos Naturais (PPGRN) da UENF; ao Instituto Chico Mendes de Conservação da Biodiversidade (ICMBio), pela permissão para realização do presente trabalho na Reserva Biológica (REBIO) União; ao Programa "Pensa Rio - Apoio ao Estudo de Temas Relevantes e Estratégicos para o Estado do Rio de Janeiro" da FAPERJ (APQ1E-26/110.400/2010), pelo auxílio financeiro ao projeto; aos técnicos Helmo Carvalho, Gerson Purificação e José Wanderley Degel, do LCA, pelo apoio no trabalho de campo; ao professor Marcelo Trindade do Nascimento, do LCA, pelo suporte logístico; ao técnico Roberto Silva do Laboratório de Fauna do Solo da Embrapa Agrobiologia, pelo suporte na identificação dos artrópodes edáficos.

\section{Referências}

ALEM, S.; PAVLIS, J. Native woody plants diversity and density under Eucalyptus camaldulensis plantation, in Gibie Valley, South Western Ethiopia. Open Journal of Forestry, Hubei Province, v. 2, n. 4, p. 232-239, 2012.

ALENCAR, A. L. et al. Regeneração natural avançada de espécies arbóreas nativas no sub-bosque de povoamentos de Eucalyptus saligna Smith., na zona da mata sul de Pernambuco. Ciência Florestal, Santa Maria, v. 21, n. 2, p. 183-192, 2011.

ALVARES, C. A. et al. Koppen's climate classification map for Brazil. Meteorologische Zeitschrift, Stuttgart, v. 22, n. 6, p. 711-728, 2013.

ASHFORD, O. S. et al. Litter manipulation and the soil arthropod community in a lowland tropical rainforest. Soil Biology \& Biochemistry, Oxford, v. 62, p. 5-12, 2013.

BULTMAN, T. L.; UETZ, G. W. Abundance and community structure of forest floor spiders following litter manipulation. Oecologia, Berlin, v. 55, p. 34-41, 1982.

CAMARA, R.; CORREIA, M. E. F.; VILLELA, D. M. Effects of eucalyptus plantations on soil arthropod communities in a Brazilian Atlantic Forest conservation unit. Bioscience Journal, Uberlândia, v. 28, n. 3, p. 445-455, 2012.

CAMARA, R. et al. Effects of natural Atlantic Forest regeneration on soil fauna, Brazil. Floresta e Ambiente, Seropédica, v. 25, n. 1, e20160017, $2018 \mathrm{a}$.

CAMARA, R. et al. Relação entre sucessão secundária, solo e serapilheira em uma Reserva Biológica no Estado do Rio de Janeiro, Brasil. Ciência Florestal, Santa Maria, v. 28, n. 1, p. 674-686, 2018 b.

CAMARA, R. et al. Atlantic Forest size effect on structure and composition of soil arthropods community, RJ, Brazil. Floresta, Curitiba, v. 47, n. 2, p. 145-156, 2017. 
CASTRO, A.; WISE, D. H. Influence of fine woody debris on spider diversity and community structure in forest leaf litter. Biodiversity and Conservation, Netherlands, v. 18, p. 3705-3731, 2009.

CIERJACKS, A. et al. Impact of sowing, canopy cover and litter on seedling dynamics of two Polylepis species at upper tree lines in central Ecuador. Journal of Tropical Ecology, Cambridge, v. 23, p. 309-318, 2007.

CUNHA NETO, F. V. et al. Soil fauna as an indicator of soil quality in forest stands, pasture and secondary forest. Revista Brasileira de Ciência do Solo, Viçosa, MG, v. 36, p. 1407-1417, 2012.

EVARISTO, V. T.; BRAGA, J. M. A.; NASCIMENTO, M. T. Atlantic Forest regeneration in abandoned plantations of eucalypt (Corymbia citriodora (Hook.) K. D. Hill and L. A. S. Johnson) in Rio de Janeiro, Brazil. Interciencia, Caracas, v. 36, n. 6, p. 431-436, 2011.

FERREIRA, C. R. et al. Edaphic arthropods in different successional stages of Atlantic forest and abandoned pasture areas. Comunicata Scientiae, Bom Jesus, v. 8, n. 2, p. 296-306, 2017.

FRASSON, J. M. F. et al. Litter decomposition of two pioneer tree species and associated soil fauna in areas reclaimed after surface coal mining in Southern Brazil. Revista Brasileira de Ciência do Solo, Viçosa, MG, v. 40, e0150444, 2016.

GAMA-RODRIGUES, E. F. et al. Alterações na biomassa e na atividade microbiana da serapilheira e do solo, em decorrência da substituição de cobertura florestal nativa por plantações de eucalipto, em diferentes sítios da região sudeste do Brasil. Revista Brasileira de Ciência do Solo, Viçosa, MG, v. 32, p. 1489-1499, 2008.

GILL, R. W. Soil microarthropod abundance following old-field litter manipulation. Ecology, New York, v. 50, n. 5 , p. $805-816,1969$.

GONÇALVES, M. F.; PEREIRA, J. A. Abundance and diversity of soil arthropods in the olive grove ecosystem. Journal of Insect Science, Oxford, v. 12, n. 20, p. 1-14, 2012.

IBÁÑEZ, I.; SCHUPP, E. W. Effects of litter, soil surface conditions, and microhabitat on Cercocarpus ledifolius Nutt. seedling emergence and establishment. Journal of Arid Environments, Oxford, v. 52, n. 2, p. 209-221, 2002.

KEELING, H. C.; PHILLIPS, O. L. A calibration method for the crown illumination index for assessing forest light environments. Forest Ecology and Management, Oxford, v. 242, n. 2/3, p. 431-437, 2007.

LIMA, K. D. R. et al. Soil fauna as bioindicator of recovery of degraded areas in the Caatinga biome. Revista Caatinga, Mossoró, v. 30, n. 2, p. 401-411, 2017.

LINDSAY, E. A.; CUNNINGHAM, S. A. Livestock grazing exclusion and microhabitat variation affect invertebrates and litter decomposition rates in woodland remnants. Forest Ecology and Management, Oxford, v. 258, p. 178-187, 2009.

MANHÃES, C. M. C. et al. Meso- and macrofauna in the soil and litter of leguminous trees in a degraded pasture in Brazil. Agroforestry Systems, [S.1.], v. 87, n. 5, p. 993-1004, 2013.

MARTIUS, C. et al. Microclimate in agroforestry systems in central Amazonia: does canopy closure matter to soil organisms? Agroforestry Systems, Netherlands, v. 60, p. 291-304, 2004.

MIRANDA, C. C.; CANELLAS, I. P.; NASCIMENTO, M. T. Caracterização da matéria orgânica do solo em fragmentos de Mata Atlântica e plantios abandonados de eucalipto, Reserva Biológica União, RJ. Revista Brasileira de Ciência do Solo, Viçosa, MG, v. 31, p. 905-916, 2007.

MORÓN-RÍOS, A.; HUERTA-LWANGA, E. Soil macrofauna of two successional evergreen cloud forest stages from the Cerro Huitepec Nature Reserve, San Cristóbal de Las Casas, Chiapas, México. Interciencia, Caracas, v. 31, n. 8, p. 611-615, 2006.

OBER, H. K.; DEGROOTE, L. W. Effects of litter removal on arthropod communities in pine plantations. Biodiversity and Conservation, Netherlands, v. 20, p. 1273-1286, 2011. 
ODUM, E. P. Ecologia. Rio de Janeiro: Guanabara Koogan, 1988. 434 p.

ONOFRE, F. F.; ENGEL, V. L.; CASSOLA, H. Regeneração natural de espécies da Mata Atlântica em sub-bosque de Eucalyptus saligna Smith. em uma antiga unidade de produção florestal no Parque das Neblinas, Bertioga, SP. Scientia Forestalis, Piracicaba, v. 38, n. 85, p. 39-52, 2010.

PEREIRA, G. H. A. et al. Decomposição da serrapilheira, diversidade e funcionalidade de invertebrados do solo em um fragmento de Floresta Atlântica. Bioscience Journal, Uberlândia, v. 29, n. 5, p. 1317-1327, 2013.

ROVEDDER, A. P. M. et al. Organismos edáficos como bioindicadores da recuperação de solos degradados por arenização no Bioma Pampa. Ciência Rural, Santa Maria, v. 39, n. 4, p. 1061-1068, 2009.

SAYER, E. J. Using experimental manipulation to assess the roles of leaf litter in the functioning of forest ecosystems. Biological Reviews, Cambridge, v. 81, p. 1-31, 2006.

SAYER, E. J. et al. Arthropod abundance and diversity in a lowland tropical forest floor in Panama: the role of habitat space vs. nutrient concentrations. Biotropica, New Jersey, v. 42, n. 2, p. 194-200, 2010.

SAYER, E. J.; TANNER, E. V. J.; LACEY, A. L. Effects of litter manipulation on early-stage decomposition and meso-arthropod abundance in a tropical moist Forest. Forest Ecology and Management, Oxford, v. 229, p. 285-293, 2006.

WANG, S.; RUAN, H.; WANG, B. Effects of soil microarthropods on plant litter decomposition across an elevation gradient in the Wuyi Mountains. Soil Biology \& Biochemistry, Oxford, v. 41, p. 891-897, 2009.

WARDLE, D. A. Impacts of disturbance on detritus food-webs in agroecosystems of contrasting tillage and weed management practices. Advances in Ecological Research, Netherlands, v. 26, p. $105-185,1995$.

WARDLE, D. A.; PARKINSON, D. Analyses of co-ocurrence in a fungal community. Mycological Research, Cambridge, v. 95, n. 4, p. 504-507, 1991.

WOLKOVICH, E. M. Nonnative grass litter enhances grazing arthropod assemblages by increasing native shrub growth. Ecology, New York, v. 91, n. 3, p. 756-766, 2010.

YANG, X.; WARREN, M.; ZOU, X. Fertilization responses of soil litter fauna and litter quantity, quality, and turnover in low and high elevation forests of Puerto Rico. Applied Soil Ecology, Oxford, v. 37, p. 63-71, 2007.

ZHANG, A.; FU, S. Allelopathic effects of eucalyptus and the establishment of mixed stands of eucalyptus and native species. Forest Ecology and Management, Oxford, v. 258, p. 1391-1396, 2009. 